\title{
Characterization of the Rho GTPase-Activating Protein RhoGAP68F
}

\author{
Minyeop Nahm and Seungbok Lee* \\ Department of Cell and Developmental Biology, Dental Research Institute, \\ Seoul National University, Seoul 110-749, Korea
}

\begin{abstract}
Rho small GTPases control multiple aspects of neuronal morphogenesis by regulating the assembly and organization of the actin cytoskeleton. Although they are negatively regulated by GTPase activating proteins (GAPs), the roles of RhoGAPs in the nervous system have not been fully investigated. Here we describe a characterization of Drosophila RhoGAP68F that is mainly expressed in the embryonic central nervous system. RNA in situ hybridization analysis showed that expression of RhoGAP68F is highly restricted to the embryonic brain and ventral nerve cord. Database search revealed that RhoGAP68F contains an N-terminal Sec14 domain and a C-terminal RhoGAP domain. Rho-GTP pull-down assay demonstrated that the RhoGAP domain of RhoGAP68F inactivates RhoA but not Rac1 or Cdc42 in HEK293 cells. In addition, expression of RhoGAP68F in NIH3T3 cells suppressed LPA-induced stress fiber formation, which is mediated by RhoA. Finally, neuronal overexpression of RhoGAP68F causes synaptic overgrowth at the larval neuromuscular junction (NMJ). Taken together, these results suggest that RhoGAP68F may play a role in synaptic growth regulation by inactivating $\mathrm{RhoA}$.
\end{abstract}

Key words: GAP, RhoGAP68F, RhoA, F-actin, nervous system, drosophila

\section{INTRODUCTION}

Rho small GTPases including RhoA, Rac1, and Cdc42 regulate multiple aspects of neuronal development including cell migration, cell polarity, axon growth and pathfinding, and dendritic elaboration (Luo, 2000; Govek et al., 2005). They function as molecular switches that shift between an active GTP-bound state and an inactive GDP-bound state (Jaffe and Hall, 2005). The active forms of Rho

\footnotetext{
*To whom correspondence should be addressed.

TEL: 82-2-740-8685, FAX: 82-2-762-2583

e-mail: seunglee@snu.ac.kr

Received January 3, 2011

Accepted for publication January 24, 2011
}

small GTPases bind their specific effector molecules to regulate F-actin and microtubule dynamics and gene expression (Jaffe and Hall, 2005). While guanine nucleotide exchange factors (GEFs) function as positive regulators of Rho small GTPases by stimulating the exchange of bound GDP for GTP, GTPase activating proteins (GAPs) negatively regulate Rho small GTPases by enhancing the intrinsic GTPase activity of Rho proteins (Luo, 2000; Moon and Zheng, 2003; Jaffe and Hall, 2005).

As suggested by the defined roles of Rho GTPases in the nervous system, various Drosophila RhoGAP proteins have been implicated in neuronal morphogenesis. For example, p190 RhoGAP has been shown to maintain axon branch stability in 
mushroom body neurons by inactivating RhoA (Billuart et al., 2001). In addition, genetic and biochemical interaction experiments suggest that CrossGAP/Vilse mediates Robo-mediated axon repulsion by the local inactivation of Rac (Lundstrom et al., 2004; Hu et al., 2005). Recently, the Cdc42selective GAP dRich has been shown to promote synaptic growth at the neuromuscular junction (NMJ) (Nahm et al., 2010), further demonstrating the significance of RhoGAP in neuronal development.

Although the Drosophila genome contains at least 20 predicted RhoGAPs (Hu et al., 2005), many of them have not been characterized in the nervous system. In this study, we identified RhoGAP68F as a RhoA-selective GAP that is highly expressed in the embryonic central nervous system (CNS). In addition, we show that overexpression of RhoGAP68F in motor neurons causes synaptic overgrowth at the larval NMJ. These results raise the possibility that RhoGAP68F may play an essential role for neuronal development by inactivating RhoA.

\section{MATERIALS AND METHODS}

\section{Cell culture}

HEK293 and NIH3T3 cells were cultured in Dulbecco's modified Eagle's medium (DMEM) containing $10 \%$ fetal bovine serum and antibiotics. These cells were transfected using Lipofectamine 2000 (Invitrogen) according to the manufacturer's instructions. To induce actin structural changes, NIH3T3 cells were serum-starved for $12 \mathrm{hr}$ and treated for $10 \mathrm{~min}$ with $20 \mathrm{ng} / \mathrm{ml}$ lysophosphatidic acid (LPA) (Sigma), $10 \mathrm{ng} / \mathrm{ml}$ PDGF (Upstate Biotechnology), or $100 \mathrm{ng} / \mathrm{ml}$ Bradykinin (Sigma) before fixation.

\section{Plasmids}

The full-length cDNA clone for RhoGAP68F was obtained from the Drosophila Genomics Resource Center. The entire coding region of RhoGAP68F was amplified by PCR using appropriate primers and introduced into $p c D N A-H A$ (Invitrogen) for mammalian expression. The RhoGAP68F- R315A point mutation was made using the QuikChange Multi kit (Stratagene). Plasmids for Myc- RhoA, Rac1, and Cdc42 were previously described ( $\mathrm{Nahm}$ et al.,
2006).

\section{RNA in situ hybridization}

RNA in situ hybridization on whole mount embryos was performed as previously described (Tautz and Pfeifle, 1989). The entire RhoGAP68F cDNA was cloned into pBluscript (Stratagene). The resulting plasmid was linearized and used for the generation of a digoxygenin (DIG)-UTP-labeled RhoGAP68F RNA probe by run-off transcription. Embryos were dechorionated and fixed in PBS containing 5\% formaldehyde. Fixed embryos were hybridized with DIG-labeled riboprobes at $62^{\circ} \mathrm{C}$ and then incubated with alkaline phosphatase-conjugated anti-DIG antibody for $1 \mathrm{hr}$ at room temperature. The alkaline phosphatase reaction was performed using 5-bromo4-chloro-indoyl phosphate (BCIP) and nitro blue tetrazolium (NBT). The embryos were mounted in Permount (Fisher Scientific).

\section{Rho-GTP pull-down assay}

Inactivation of Rho proteins by RhoGAP68F was analyzed using the EZ-detect Rho, Rac, or Cdc42 activation kit (Pierce), as previously described (Nahm et al., 2006). Briefly, HEK293 cells transiently expressing $p C M V-M y c-R h o A$, Rac1, or Cdc42 and pcDNA-HA-RhoGAP68F or pcDNA-HA-RhoGAP68F$R 315 A$ were lysed in $25 \mathrm{mM}$ Tris $(\mathrm{pH} 7.5), 150 \mathrm{mM}$ $\mathrm{NaCl}, 5 \mathrm{mM} \mathrm{MgCl}$, $1 \% \mathrm{NP}-40,1 \mathrm{mM}$ DTT, and $5 \%$ glycerol. Cell lysates were clarified by centrifugation at $13,000 \times \mathrm{g}$ for $10 \mathrm{~min}$. Equal volumes of lysates were then incubated at $4^{\circ} \mathrm{C}$ for $1 \mathrm{hr}$ with glutathione-Sepharose 4B-bound GST-Rhotekin-PBD (for RhoA-GTP) or GST-PAK1-PBD (for Rac1-GTP and Cdc42-GTP) fusion protein. The beads were washed four times with the lysis buffer and boiled in SDS sample buffer. The amount of GTP-bound Myc-RhoA, Rac1, or Cdc42 was determined by western blotting using an anti-Myc antibody (Cell Signaling). The total amounts of Rho proteins from cell lysates were determined by western blotting using an antiMyc or anti-HA (Roche) antibody.

\section{Fly stock and genetics}

The wild-type strain used in this study was $w^{1118}$. A transgenic line carrying UAS-RhoGAP68F was obtained in the $w^{1118}$ background using standard protocols (Robertson et al., 1988). Elav-GeneSwitch- 
GAL4 (Osterwalder et al., 2001) was used to drive UAS-RhoGAP68F expression in postembryonic neurons.

\section{Immunohistochemistry}

NIH3T3 cells were transfected with a plasmid encoding HA-RhoGAP68F or HA-RhoGAP68F-R315A. Immunostaining of transfected cells was performed as previously described (Nahm et al., 2006). Filamentous actin structures were visualized using rhodamine-conjugated phalloidin (Invitrogen) at 1:250.

Larval body wall muscles were dissected from wandering third-instar larvae in $\mathrm{Ca}^{2+}$-free $\mathrm{HL3}$ saline (Stewart et al., 1994) and fixed in $4 \%$ formaldehyde in PBS for 30 min. Fixed muscles were washed with PBT (PBS, 0.1\% Triton X-100) and incubated with goat anti-HRP conjugated with FITC (Jackson ImmunoResearch, $1: 200$ ) for $1 \mathrm{hr}$ at room temperature. Image acquisition and morphological quantification were performed as previously described (Nahm et al., 2010).

\section{RESULTS AND DISCUSSION}

\section{Identification of RhoGAP68F, a RhoA-specific GAP that is highly expressed in the nervous system}

The Drosophila genome contains at least 20 RhoGAPs for the Rho family (Hu et al., 2005); however, their role(s) in the developing nervous system are still poorly understood. As an initial step to address this issue, we searched by RNA in situ hybridization on whole-mount embryos for previously uncharacterized RhoGAP genes expressed in the central nerves system (CNS), and found that the annotated gene RhoGAP68F (CG6811) fulfills the criterion. A high level of RhoGAP68F message was uniformly detected in early stage embryos (e.g. stage 5; Fig. 1A), suggesting a significant maternal contribution (Fig. 1A). RhoGAP68F expression was restricted to brain lobes and the ventral nerve cord by stage 13 (Fig. 1B). The enrichment of RhoGAP68F expression in the embryonic CNS was maintained until the end of embryogenesis (Fig. 1C).

The Flybase database predicts that RhoGAP68F encodes a 476-amino acid protein consisting of an $\mathrm{N}$-terminal Sec14 domain (amino acids 95-241) and a C-terminal RhoGAP domain (amino acids 289-

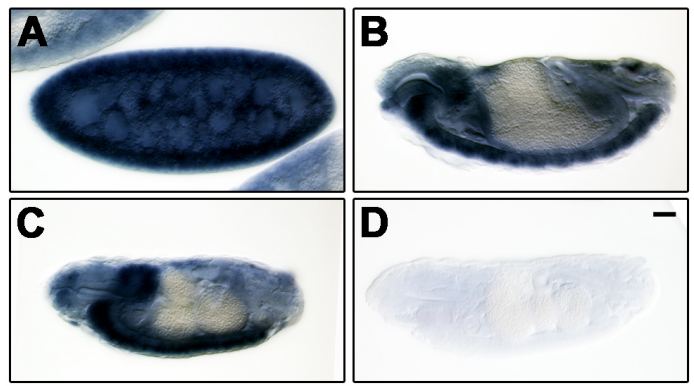

Fig. 1. Expression of RhoGAP68F transcript during embryo development. In situ hybridization on whole-mount embryos was performed to using RhoGAP68F antisense RNA probe. All embryos are anterior to the left, and dorsal is up. (A) Lateral view of a stage 5 embryo. RhoGAP68F mRNA was broadly detected throughout the entire embryo, indicating its maternal loading. (B) Lateral view of a stage 13 embryo. RhoGAP68F mRNA was abundant in the developing CNS. (C) Lateral view of a stage 16 embryo. High levels of RhoGAP68F expression were maintained in the CNS. (D) Lateral view of a stage 16 embryo. A RhoGAP68F sense probe shows no signal. Scale bar, $20 \mu \mathrm{m}$.

461). This domain organization is identical to the human p50RhoGAP protein (Fig. 2A). Overall, the RhoGAP68F protein displays $37 \%$ identity and $60 \%$ similarity to its human counterpart at the amino acid level. A previous work has shown that RhoGAP68F has GAP activity toward human RhoA in vitro (Sanny et al., 2006). To examine the in vivo substrate of the RhoGAP68F, HEK293 cells were transiently transfected with $p C M V-M y c-R h o A$, Rac1, or Cdc42 along with either pcDNA-HA or pcDNA$H A-R h o G A P 68 F$, and the amounts of active, GTPbound Rho GTPases in the lysates of the transfectants were determined by p21-binding domain (PBD) pull-down assays (see Materials and Methods). As shown in Fig. 2B, expression of HA-RhoGAP68F efficiently removed GTP-RhoA (Fig. 2B). However, the level of GTP-Rac1 or GTP-Cdc42 was not changed by HA-RhoGAP68F expression (Fig. 2B), suggesting that RhoGAP68F acts as a RhoAselective GAP in vivo. RhoGAP68F contains the arginine finger at position 315 , which is conserved in RhoGAP-family proteins. A RhoGAP68F mutant carrying a substitution mutation of this amino acid (RhoGAP68F-R315A) did not effectively reduce the level of GTP-RhoA (Fig. 2B).

\section{RhoGAP68F inhibits LPA-induced stress fiber formation mediated by RhoA}

Rho small GTPases are well known for their 
A

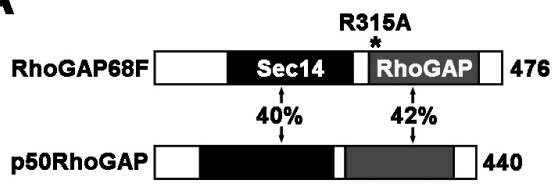

B

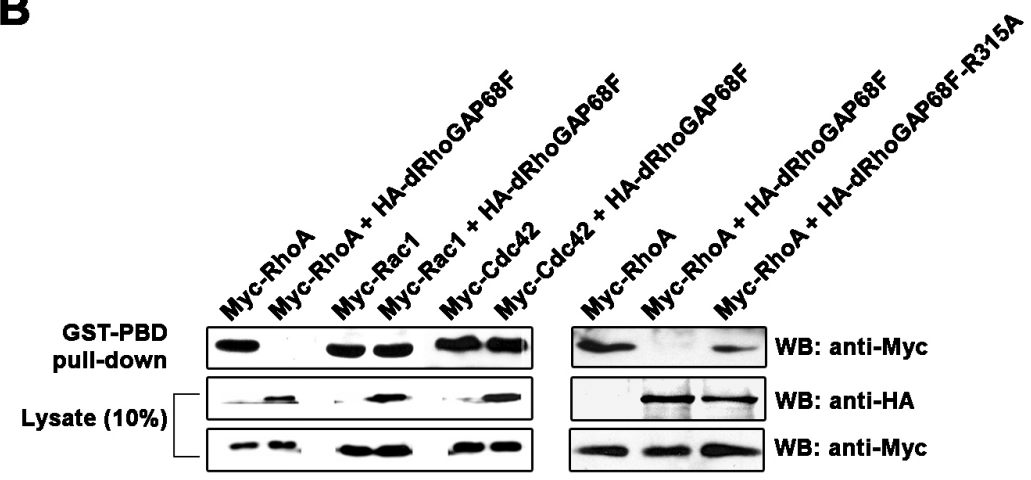

Fig. 2. RhoGAP68F is a RhoA-selective GAP. (A) Domain structure of RhoGAP68F and human p50RhoGAP. The percent amino acid identity between the homologs is indicated. The point mutation R315A in the GAP domain of RhoGAP68F is marked by asterisk. (B) RhoGAP68F inactivates RhoA but not Rac1 or Cdc42 in cells. HEK293 cells were co- transfected with a plasmid encoding Myc-RhoA, Rac1, or Cdc42 along with a plasmid encoding HA, HA-RhoGAP68F, or HA-RhoGAP68FR315A. Following transfection, GTP-loaded RhoA, Rac1, and Cdc42 were precipitated with GST fusion PBDs of Rhotekin (for RhoA) and PAK1 (for Rac1 and Cdc42). The levels of GTP-bound GTPases in the precipitates were determined by western blot analysis using an anti-Myc antibody (upper panel). Levels of HA and Myc proteins in the cell lysates were determined by western blot analysis using anti-HA (middle panel) and anti-Myc (lower panel) antibodies, respectively. specific effects on the organization of the actin cytoskeleton in fibroblasts: RhoA produces stress fibers associated with focal adhesion complexes, Rac1 induces lamellipodia and membrane ruffles, and Cdc42 triggers the formation of filopodia or microspikes (Hall, 1998). To further characterize the substrate specificity of RhoGAP68F, we examined the effect of RhoGAP68F overexpression on the actin cytoskeleton in cultured cells. NIH3T3 cells were transfected with HA-tagged RhoGAP68F and then stained with rhodamine-conjugated phalloidin to visualize actin filaments. As shown in Fig. 3, expression of RhoGAP68F inhibited lysophosphatidic acid (LPA)-induced stress fiber formation mediated by RhoA (Fig. 3A). However, RhoGAP68F expression had no effect on either PDGF-induced membrane ruffling mediated by Rac1 or Bradykinin-induced filopodia formation mediated by Cdc42. Finally, LPAinduced stress fiber formation was not significantly affected by expression of the R315A GAP-deficient mutant (Fig. 3B). These results suggest that RhoGAP68F acts as a RhoA-selective GAP in NIH3T3 cells.

\section{Neuronal overexpression of RhoGAP68F induces synaptic overgrowth at the Drosophila larval neuromuscular junction}

Because RhoGAP68F was found highly in the embryonic CNS, we wished to determine the role of RhoGAP68F in synapse development. To investigate this, we expressed exogenous RhoGAP68F in all
A
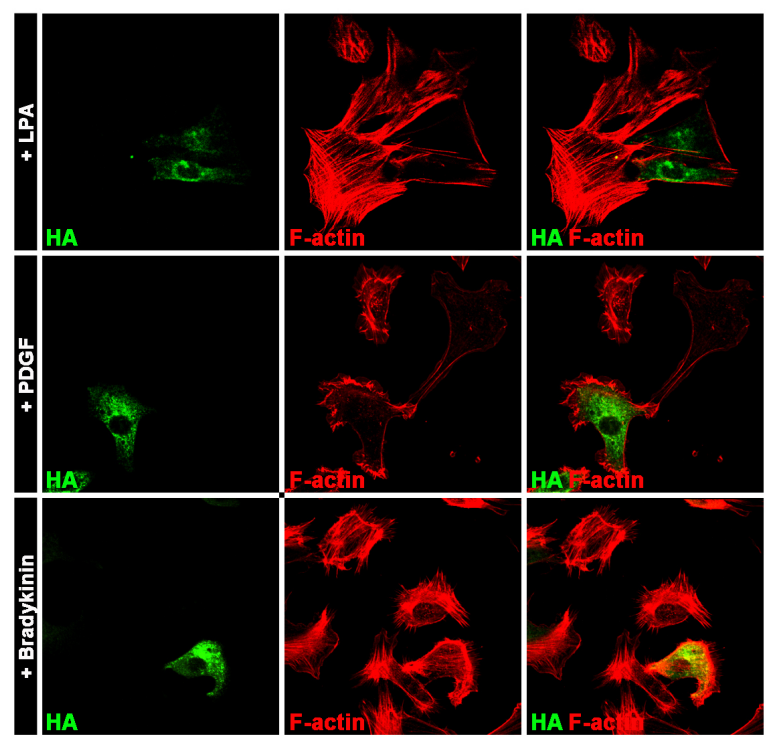

B
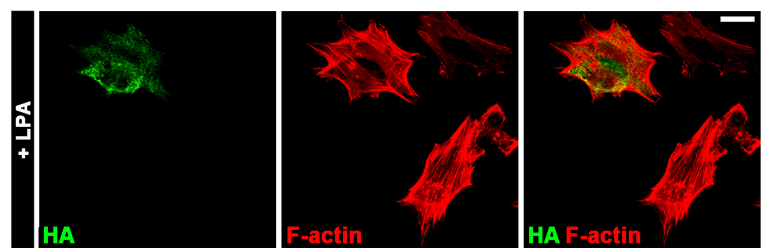

Fig. 3. Effect of RhoGAP68F on actin cytoskeletal changes induced by LPA, PDGF, and Bradykinin in NIH3T3 cells. NIH3T3 cells were transfected with a plasmid expressing either HARhoGAP68F (A) or HA-RhoGAP68F-R315A (B). After a $12 \mathrm{hr}$ serum withdrawal, cells were incubated with $20 \mathrm{ng} / \mathrm{ml}$ LPA for RhoA activation, $10 \mathrm{ng} / \mathrm{ml}$ PDGF for Rac1 activation, or 100 $\mathrm{ng} / \mathrm{ml}$ Bradykinin for Cdc42 activation. Cells were stained with anti-HA and rhodamine-conjugated phalloidin to visualize HARhoGAP68F (green) and filamentous actin (red), respectively. Scale bar, $20 \mu \mathrm{m}$. 


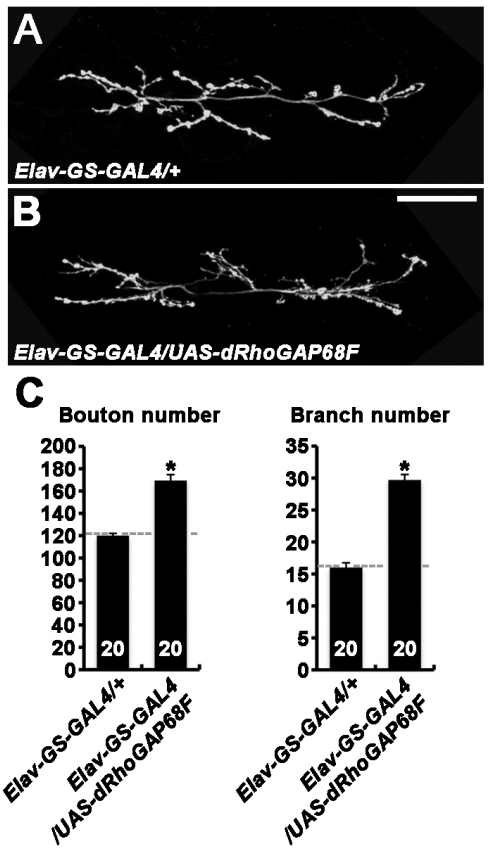

Fig. 4. Neuronal overexpression of RhoGAP68F causes synaptic overgrowth at the larval NMJ. (A, B) Confocal images of third instar NMJs 6/7 labeled with anti-HRP are shown for Elav-GSGAL4/+ and Elav-GS-GAL4/UAS-RhoGAP68F larvae. Note that presynaptic overexpression of RhoGAP68F causes NMJ overgrowth. Scale bar, $50 \mu \mathrm{m}$. (C) Quantification of total bouton number and branch number at NMJs $6 / 7$ in Elav-GS-GAL4/+ and Elav-GS-GAL4/UAS-RhoGAP68F third instar larvae. Data are mean \pm SEM. The numbers of NMJs analyzed are indicated inside bars. Statistically significant differences are indicated by asterisk $(p<0.001)$.

neurons and then examined synaptic growth at the larval neuromuscular junction (NMJ). Overexpression of RhoGAPs at embryonic stages could cause defects in axon development (Billuart et al., 2001), complicating analysis of its effect on synaptic growth. Therefore, we overexpressed UAS-RhoGAP68F in all neurons from the early first instar stage, when synapse formation between motor axons and target muscles is already finished, by employing the inducible neuronal GAL4 driver Elav-GS-GAL4 system, in which gene expression is induced only by the addition of the progesterone analog RU486 (Osterwalder et al., 2001). When raised on the food containing RU486 throughout larval development, third instar Elav-GS-GAL4/UAS-RhoGAP68F animals displayed excessive NMJ growth (Fig. 4A, B). To quantify this phenotype, we measured bouton number and branch number at NMJ 6/7 in abdominal segment 2 (Fig. 4C). Bouton number was increased by $42 \%$ in Elav- GS-GAL4/UAS-RhoGAP68F larvae compared with Elav-GS-GAL4/+ controls (169.4 \pm 5.3 versus $120.0 \pm 2.1$; mean $\pm S E M ; p<0.001$ ). In addition, the number of NMJ branches was increased by $86 \%$ (29.7 \pm 0.8 versus $16.0 \pm 0.7 ; p<0.001)$, supporting a novel role for RhoGAP68F in the regulation of synaptic growth.

In summary, we provide evidence that RhoGAP68F functions as a negative regulator of RhoA signaling in vivo. This conclusion is consistent with a previous finding that RhoGAP68F acts as a RhoAselective GAP in vitro (Sanny et al., 2006). Although RhoGAP68F is shown to regulate morphogenetic events during gastrulation of the embryo (Sanny et al., 2006), it has not been addressed whether they also regulates neuronal morphogenesis. In this study, we have shown that RhoGAP68F is highly expressed in the nervous system and that presynaptic overexpression of RhoGAP68F affects NMJ growth, raising the possibility that RhoA signaling and its regulators may play a key role in synaptic growth and plasticity during development.

\section{ACKNOWLEDGEMENTS}

This work was supported by a grant of the Korea Healthcare Technology R\&D Project, Ministry for Health, Welfare and Family Affairs, Republic of Korea (A084972).

\section{REFERENCES}

Billuart P, Winter CG, Maresh A, Zhao X and Luo L (2001) Regulating axon branch stability: the role of p190 RhoGAP in repressing a retraction signaling pathway. Cell 107:195-207.

Govek EE, Newey SE and Van Aelst L (2005) The role of the Rho GTPases in neuronal development. Genes Dev 19:1-49.

Hall A (1998) Rho GTPases and the actin cytoskeleton. Science 279:509-514.

Hu H, Li M, Labrador JP, McEwen J, Lai EC, Goodman CS and Bashaw GJ (2005) Cross GTPase-activating protein (CrossGAP)/Vilse links the Roundabout receptor to Rac to regulate midline repulsion. Proc Natl Acad Sci USA 102:4613-4618.

Jaffe $A B$ and Hall A (2005) Rho GTPases: biochemistry and biology. Annu Rev Cell Dev Biol 21:247-269.

Lundstrom A, Gallio M, Englund C, Steneberg P, Hemphala J, Aspenstrom P, Keleman K, Falileeva L, Dickson BJ and Samakovlis C (2004) Vilse, a conserved Rac/Cdc42 GAP mediating Robo repulsion in tracheal cells and axons. 
Genes Dev 18:2161-2171.

Luo L (2000) Rho GTPases in neuronal morphogenesis. Nat Rev Neurosci 1:173-180.

Moon SY and Zheng Y (2003) Rho GTPase-activating proteins in cell regulation. Trends Cell Biol 13:13-22.

Nahm M, Kim S, Paik SK, Lee M, Lee S, Lee ZH, Kim J, Lee D, Bae YC and Lee S (2010) dCIP4 (Drosophila Cdc42-interacting protein 4) restrains synaptic growth by inhibiting the secretion of the retrograde Glass bottom boat signal. J Neurosci 30:8138-8150.

Nahm M, Lee M, Baek SH, Yoon JH, Kim HH, Lee ZH and Lee S (2006) Drosophila RhoGEF4 encodes a novel RhoA-specific guanine exchange factor that is highly expressed in the embryonic central nervous system. Gene 384:139-144.

Nahm M, Long AA, Paik SK, Kim S, Bae YC, Broadie K and Lee $S$ (2010) The Cdc42-selective GAP Rich regulates postsynaptic development and retrograde BMP transsynaptic signaling. J Cell Biol 191:661-675.

Osterwalder T, Yoon KS, White BH and Keshishian H (2001)
A conditional tissue-specific transgene expression system using inducible GAL4. Proc Natl Acad Sci USA 98:1259612601.

Robertson HM, Preston CR, Phillis RW, Johnson-Schlitz DM, Benz WK and Engels WR (1988) A stable genomic source of $\mathrm{P}$ element transposase in Drosophila melanogaster. Genetics 118:461-470.

Sanny J, Chui V, Langmann C, Pereira C, Zahedi B and Harden N (2006) Drosophila RhoGAP68F is a putative GTPase activating protein for RhoA participating in gastrulation. Dev Genes Evol 216:543-550.

Stewart BA, Atwood HL, Renger JJ, Wang J and Wu CF (1994) Improved stability of Drosophila larval neuromuscular preparations in haemolymph-like physiological solutions. J Comp Physiol A 175:179-191.

Tautz D and Pfeifle C (1989) A non-radioactive in situ hybridization method for the localization of specific RNAs in Drosophila embryos reveals translational control of the segmentation gene hunchback. Chromosoma 98:81-85. 